\title{
Bioleaching of Precious Metals from an Oil-Fired Ash Using Organic Acids Produced by Aspergillus Nigerin Shake Flasks and Bioreactor
}

\author{
Payam Rasoulnia and Seyyed Mohammad Mousavi
}

\begin{abstract}
In the present study, bioleaching of vanadium and nickel from an oil-fired ash sample was conducted using Aspergillus niger fungus. Oil-fired ash is a major by-product of thermal power plants which is considered as a secondary source for $\mathrm{V}$ and Ni recovery. The experiments were carried out using spent-medium bioleaching method in both Shake flasks and also bubble column bioreactor, in order to compare them together. In Shake flask experiments the fungus was cultured for 14 days, where the maximum production of organic acids was observed, while in bubble column bioreactor experiments a 7 days fermentation period was applied. Measurement of produced organic acids during fermentation period indicated that the main excreted metabolites by Aspergillus niger in Shake flasks were different with that of bioreactor since the fermentation conditions in these two various scales differed significantly. In both of the scales the concentrations of citric, oxalic, gluconic and malic acids were measured and it was revealed that in Shake flask experiments citric acid, and in bubble column bioreactor oxalic acid was the major lixiviant, while the production of gluconic acid was similarly lower. In Shake flask and during 14 days of fermentation of Aspergillus niger, 8080 ppm citric acid and $1170 \mathrm{ppm}$ oxalic acid was produced, while in bubble column bioreactor and over 7 days of fungal growth, 17185 ppm oxalic acid and 1040 ppm citric acid was secreted. For conducting the leaching tests using the spent-media obtained from both of fermentation experiments, a $60{ }^{\circ} \mathrm{C}$ leaching temperature, 7 days leaching duration and solid to liquid ratio of $3 \%(\mathrm{w} / \mathrm{v})$ was selected. Using Shake flask experiments spent-media, maximum $\mathrm{V}$ and $\mathrm{Ni}$ recovery yields were $97 \%$ and $50 \%$ respectively, whereas using bubble column bioreactor spent-medium, $100 \%$ of $\mathrm{V}$ and $33 \%$ of $\mathrm{Ni}$ was recovered.
\end{abstract}

Index Terms-Aspergillus niger, Bubble column bioreactor, Oil-fired ash, Spent-medium bioleaching.

\section{INTRODUCTION}

Power plants firing heavy fuel oil produce huge amounts of ashes as solid wastes, which seriously need to be managed and processed. Recycling precious metals of $\mathrm{V}$ and $\mathrm{Ni}$ from these oil-fired ashes which are considered as secondary sources of metals recovery, not only has a great economic importance for use in industry, but also it is noteworthy from the environmental point of view [1], [2]. Vanadium is an important metal that is mainly used in steel industry because of its physical properties of hardness, tensile strength, and

Manuscript received April 18, 2016; revised August 15, 2016.

The authors are with Biotechnology Group, Chemical Engineering Department, Tarbiat Modares University, Tehran, Iran (corresponding author: Seyyed Mohammad Mousavi; phone: +98-21-82884917; fax: +98-21-82884931; e-mail: payam.rasoulnia@gmail.com, mousavi_m@modares.ac.ir) fatigue resistance [3]. It is also utilized in oxidation catalysts, titanium-aluminum alloys and vanadium redox batteries [4] Hydrometallurgy and pyrometallurgy are two common technologies for recovery of metals from solid wastes. In hydrometallurgy metals are leached using an acid or base; whereas in pyrometallurgy metals are extracted through a heat treatment such as roasting or smelting [5], [6]. Bioleaching is a new method based on the ability of microorganisms in producing organic or inorganic acids which form extractable and soluble compounds with the metals that can be recovered. Chemolithotrophic and heterotrophic bacteria such as Acidithiobacillus sp., Acidophilum sp., Pseudomonas sp., Acetobacter sp., Arthrobactor sp. and Bacillus sp.are mainlyused in bioleaching studies. Also heterotrophic fungi such as Aspergillus and Penicilliums species have shown a great potential in leaching of metals due to their high ability in producing organic acids [7], [8].

Biological leaching processes have some advantages in comparison to the conventional leaching techniques, including lower cost and energy consumption, more environmentally friendly and operational flexibility [6], [9]. Microbial solubilization occurs based on two different mechanisms including: direct leaching and indirect leaching mechanism. Direct leaching needs physical contact between the microbial cell wall and the surface of the solid waste and consequently metal dissolution occurs at the interface. Among different methods of bioleaching (one-step, two-step and spent-medium bioleaching), spent-medium bioleaching is considered as a more appropriate and capable method for industrial application. In this method metal dissolution occurs based on indirect leaching mechanism in which the fungus is not in direct contact with the solid waste and fungal growth is not inhibited by the toxic metals released into the medium. Therefore, formation of organic acids is enhanced and higher pulp densities of the solid waste can be applied [10], [11], [12]. Although in recent years numerous studies have been conducted on bioleaching of metals from various solid wastes, but still there is a lack of sufficient research on spent-medium bioleaching method efficiency and its application in a semi-industrial scale. Therefore, in the present study, Aspergillus niger potential in recovery of precious metals of $\mathrm{V}$ and $\mathrm{Ni}$ from an oil-fired ash sample using spent-medium bioleaching method and in two different scales was investigated. In order to scale up the process and compare the results, along with the shake flask studies, bubble column bioreactor experiments were also carried out. Use of a bioreactor is a crucial step in developing a process form experimental surveys to industrial schemes [6]. 


\section{MATERIALS AND METHODOLOGY}

Oil-fired ash: The oil-fired ash sample was provided by a thermal power plant in Tehran, Iran.A household mill and a vibrator shifter were used for grinding and screening the sample to particle sizes of less than $75 \mu \mathrm{m}$. To determine the metal content of oil-fired ash, X-ray fluorescence (XRF; PW2404, Philips, Netherland) analysis was applied. The metal phases present in the solid waste were determined using X-ray diffraction (XRD; X'Pert MPD, Philips, Netherland) analysis.

Microorganism: Aspergillus nigerfungus (PTCC 5210) was purchased from the Iranian Research Organization for Science and Technology (IROST).Prior to fungal inoculum preparation and bioleaching experiments, seven days old cultures of the fungus on Petri dishes containing potato dextrose agar (PDA) were provided.

Fungal inoculum preparation: In order to prepare fungal inoculum, the spores were collected from the surface of PDA plates using sterilized distilled water and then the number of spores was adjusted to $10^{7}$ spores under an optical microscope using a Thoma chamber. Finally proportional to the volume of prepared medium, a certain percentage of the inoculum was inoculated into the shake flasks and also the bubble column bioreactor.

Measurement of produced organic acids: in order to measure the concentration of excreted organic acids (oxalic, citric, gluconic and malic acids), High Performance liquid chromatography (HPLC; Sykam, MACHERY-NAGEL, Germany) analysis was used. For detection of organic acids concentrations using a DAD UV-Vis detector, $20 \mu \mathrm{l}$ of HPLC-grade solutions of organic acids, as standards, were injected into a $250 \times 4.6 \mathrm{~mm}$, Nucleodur $\mathrm{C} 18 \mathrm{ec}, 5 \mu \mathrm{m}$ column.

Bubble column bioreactor: Dimensional characteristics of the bubble column bioreactor used in this study include: a column height of $55 \mathrm{~cm}$, inner diameter of $7 / 5 \mathrm{~cm}$ and working volume of $1 \mathrm{~L}$. To maintain the temperature of bioreactor at $30{ }^{\circ} \mathrm{C}$, water was heated to the desired temperature in a bath, pumped into the jacket around the bioreactor and circulated. The required air for sufficient aeration of the culture was provided by a compressor (HAILEA, China). After adjustment of the air flow at the desiredrateusing a rotameter (INSTRUMENTS INC DWYER, USA), sterilized air was distributed through the medium via a spargerat the bottom of bubble column bioreactor.

Bioleaching experiments: In spent-medium bioleaching, Aspergillus niger was initially cultured in sucrose medium consisting of $100 \mathrm{~g} / \mathrm{l}$ sucrose, $1.5 \mathrm{~g} / 1 \mathrm{NaNO}_{3}, 0.5 \mathrm{~g} / 1 \mathrm{KH}_{2} \mathrm{PO}_{4}$, $0.025 \mathrm{~g} / \mathrm{l} \mathrm{MgSO}{ }_{4} .7 \mathrm{H}_{2} \mathrm{O}, 0.025 \mathrm{~g} / \mathrm{KCl}$ and $1.6 \mathrm{~g} / \mathrm{l}$ yeast extract. After determining the optimum fungal growth period through measurement of produced organic acids using High Performance Liquid Chromatography analysis, the biomass was separated from the culture and the oil-fired ash was added to the spent-medium containing maximum concentration of organic acids. The fungal cultures in Shake flasks were kept in a $30^{\circ} \mathrm{C}$ incubator, at $130 \mathrm{rpm}$, over 14 days. The fermentation period in bubble column bioreactor was 7 days, at $30{ }^{\circ} \mathrm{C}$ with an aeration rate of 760 $(\mathrm{ml} / \mathrm{min})$.The leaching tests using the spent-media obtained from the fermentation of Aspergillus nigerwere conducted at temperature of $60{ }^{\circ} \mathrm{C}$ and over 7 days. All the experiments were carried out under sterilized conditions.

\section{RESULTS AND DISCUSSION}

Oil-fired ash composition: XRF analysis of the sample revealed that the oil-fired ash comprised of vanadium, nickel and iron as the major elements, however some other metals such as $\mathrm{Cu}, \mathrm{Al}, \mathrm{Mg}$ and $\mathrm{Si}$ and also significant quantities of carbon and sulfur were present. $\mathrm{V}, \mathrm{Ni}$ and Fe respectively made up $30.5(\mathrm{~g} / \mathrm{kg}), 19.7(\mathrm{~g} / \mathrm{kg})$ and $218.8(\mathrm{~g} / \mathrm{kg})$ of the weight of oil-fired ash.

Effect of initial $\mathrm{pH}$ on Aspergillus niger growth: In order to investigate the effect of initial $\mathrm{pH}$ on fungal growth, $\mathrm{pH}$ of PDA plates were adjusted in the range of 2 to 11 and fungal colony diameter was measured during 7 days of incubation. As it is obvious from Fig. 1, fungal colony diameter in the $\mathrm{pH}$ range of 3 to 9 was approximately equal to that of control one (the plate without $\mathrm{pH}$ adjustment), suggesting that fungal growth is not inhibited in this $\mathrm{pH}$ range. But in PDA plates with $\mathrm{pH}$ values of 2,10 and 11 , growth inhibition was more considerable. Considering these results, since during fungal growth in sucrose medium, $\mathrm{pH}$ decreases from 6 in the first day of inoculation to minimum value of about 3 after completion of fungal growth, and within this range, $\mathrm{pH}$ is not considered as an inhibitory factor, initial adjustment of medium $\mathrm{pH}$ did not seem necessary.

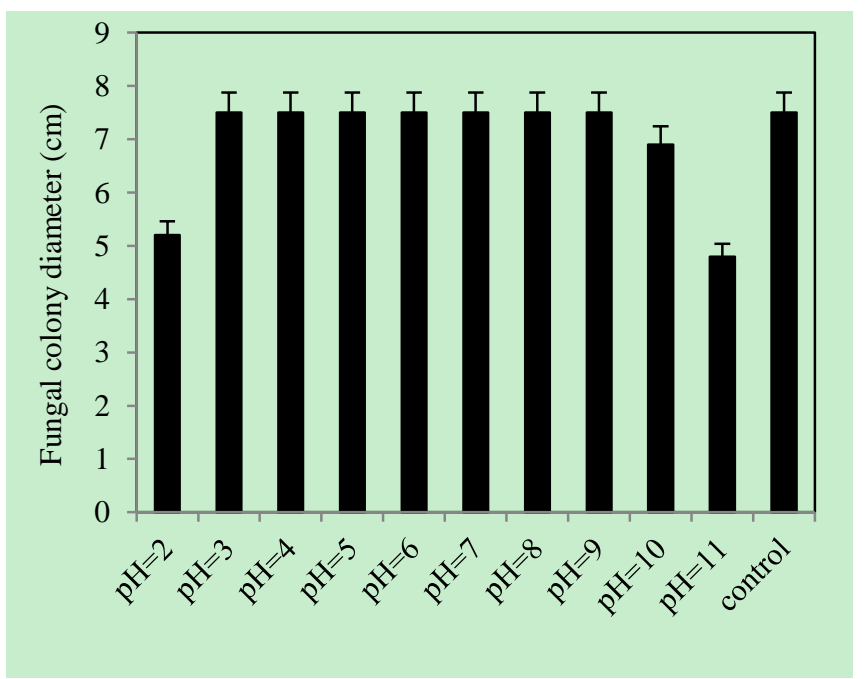

Fig. 1. Effect of initial $\mathrm{pH}$ on fungal growth.

Spent-medium bioleaching of oil-fired ash: There are three main methods for bioleaching of metals from different solid wastes which include: (a) one-step bioleaching, where solid waste addition to the bioleaching medium and inoculation are conducted simultaneously; (b) two-step bioleaching, where the addition of solid waste to the medium is done when the fungus in the starting phase of growth; and (c) spent-medium bioleaching, in which the sample is added to the cell-free medium containing maximum amount of organic acids. In spent-medium bioleaching, the solid waste is not in direct contact with the fungus, consequently the fungal growth is not retarded, and maximum organic acid is produced. In this method, the metals are leached through biogenic produced organic acids present in the medium. In shake flask experiments, firstly through measuring fungal growth characteristics including biomass dry weight, $\mathrm{pH}$ and organic 
acids concentrations, an optimal fermentation period was determined. The results indicated that Aspergillus niger decreased the medium $\mathrm{pH}$ from about 6 to 2.8 within 14 days of growth, whereas continuing incubation caused a slight increase in $\mathrm{pH}$ so that it reached around 4 at the end of day 30. The decrease in $\mathrm{pH}$ was accompanied with an increase in biomass dry weight and organic acids concentrations. Measurement of produced organic acids revealed that over 14 days of fungal growth, maximum production of organic acids was observed. In bubble column bioreactor experiments, the fermentation period was 7 days, because in longer fermentation periods due to the high volume of production of fungal biomass, aeration encountered some difficulties. The HPLC results showed that depending on the fermentation period and the scale of experiments, the fungus has different major lixiviants. In Shake flask experiments, citric acid was the main produced organic acid by the fungus and the other organic acids including gluconic, oxalic and malic acids were excreted in much lower amounts, while in bubble column bioreactor oxalic acid was the main lixiviant and its production was considerably high. In Shake flask 8080 ppm citric acid and $1170 \mathrm{ppm}$ oxalic acid was produced, while in bubble column bioreactor 17185 ppm oxalic acid and 1040 ppm citric acid was secreted. Since formation of oxalic acid occurs during the exponential growth phase and it is accelerated at $\mathrm{pH}$ values of 4 or above, in bioreactor where fermentation period was shorter and the medium $\mathrm{pH}$ was higher, oxalic acid was the main lixiviant. Citric acid is excreted mostly during a secondary production phase that is characterized by a low extracellular $\mathrm{pH}$ and a reduced growth rate. Citric acid formation in Aspergillus niger mainly occurs through glycolysis with subsequent condensation of a $\mathrm{C}_{4}$ unit with a $\mathrm{C}_{2}$ moiety. Briefly biosynthesis of citric acid includes sugar substrate uptake, glycolytic catabolism of glucose to 2 moles of pyruvate, subsequent conversion of them to oxaloacetate and acetyl-CoA, condensation of these precursors to citric acid and finally secretion of citric acid from mitochondria and mycelia respectively. In Shake flask experiments since the fermentation period was longer and the medium $\mathrm{pH}$ remained at lower values for an adequate duration, citric acid was the major organic acid produced by Aspergillus niger. Maintenance of $\mathrm{pH}$ at a low value is necessary for maximum production of citric acid [13]-[15]. Gluconic acid formation in both Erlenmeyer flasks and bubble column bioreactor experiments was almost similar. In Shake flask experiments about 2100 ppm and in bubble column bioreactor approximately 4500 ppm gluconic acid was produced. Gluconic acid is produced through conversion of significant amounts of glucose by glucose oxidase enzyme. Formation of this enzyme occurs during the starting phase of fermentation and due to the extracellular location of the enzyme, it is directly influenced by the medium $\mathrm{pH}$ and will be inactivated at $\mathrm{pH}$ values of less than 3.5[15].

The leaching tests using the spent-media obtained from the fermentation experiments in both of the scales, were performed at the same conditions of leaching duration of 7 days, leaching temperature of $60{ }^{\circ} \mathrm{C}$ and pulp density of $3 \%$ $(\mathrm{w} / \mathrm{v})$. The results revealed that in Shake flask experiments $97 \%$ of $\mathrm{V}$ and $50 \%$ of $\mathrm{Ni}$ were extracted while using bubble column bioreactor spent medium, $\mathrm{V}$ and $\mathrm{Ni}$ recoveries were $100 \%$ and $33 \%$ respectively. The lower $\mathrm{Ni}$ recovery in bioreactor is probably because of higher concentration of oxalic acid in its spent-medium and formation of insoluble nickel oxalate complex which acts as an inhibitor of the reaction, slowing down the rate of metal dissolution [16]. Organic acids, mainly citric and oxalic acids are common metabolites excreted by various fungi which their production is associated with dissolution of insoluble metal compounds. They may also cause immobilization of potentially toxic metals via formation of insoluble compounds like metal-oxalate complexes [17]. The obtained recovery yields in both different scales have been comparatively presented in Fig. 2.

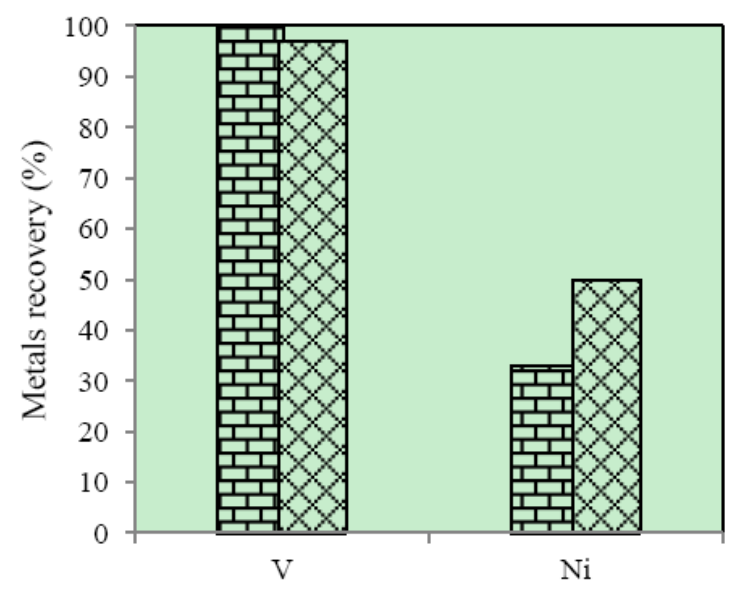

$\hookrightarrow$ Bubble column bioreactor $\square$ Shake flask

Fig. 2. Comparison of metal recovery yields using Bubble column bioreactor and shake flask spent-media.

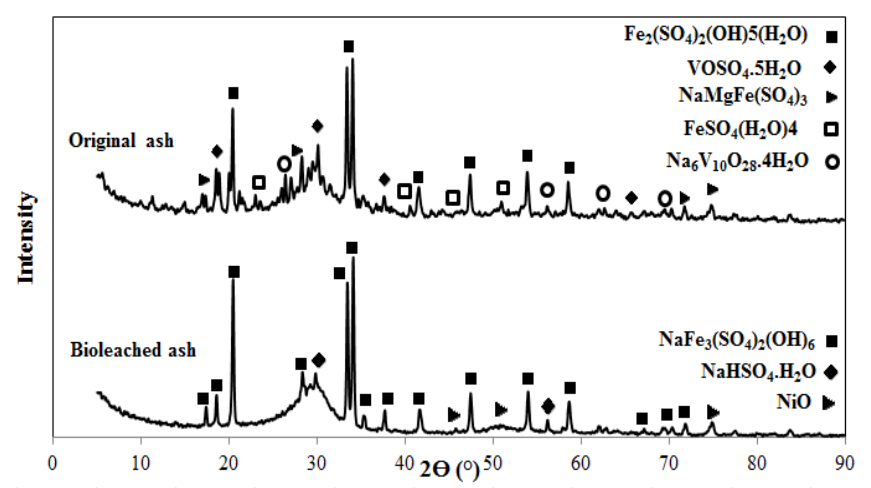

Fig. 3. XRD pattern of oil-fired ash before and after bioleaching.

XRD analysis: Fig. 3 shows XRD pattern of oil-fired ash before and after bioleaching. In original oil-fired ash vanadium was present as $\mathrm{VOSO}_{4} \cdot 5 \mathrm{H}_{2} \mathrm{O}$ and $\mathrm{Na}_{6} \mathrm{~V}_{10} \mathrm{O}_{28} .4 \mathrm{H}_{2} \mathrm{O}$ whereas after bioleaching no vanadium compound was observed. Also $\mathrm{FeSO}_{4} \cdot 4\left(\mathrm{H}_{2} \mathrm{O}\right), \quad \mathrm{NaMgFe}\left(\mathrm{SO}_{4}\right)_{3}$, $\mathrm{Fe}_{2}\left(\mathrm{SO}_{4}\right)_{2}(\mathrm{OH}) .5\left(\mathrm{H}_{2} \mathrm{O}\right)$ compounds were present in original ash but after bioleaching only $\mathrm{NaFe}_{3}\left(\mathrm{SO}_{4}\right)_{2}(\mathrm{OH})_{6}$ compound existed.

Surface morphology investigation: In order to investigate microstructural changes in surface morphology of oil-fired ash caused by biogenic produced organic acids present in spent-medium, field emission scanning electron microscopy (FE-SEM; HITACHI S-4160, Japan) analysis was used. FE-SEM images of the oil-fired ash sample before and after bioleaching have been illustrated in Fig. 4(a) and (b) respectively. The attack of fungal metabolites to the surface of oil-fired ash and formation of metal-acid complexes and consequently dissolution of the metals, have caused an 
increase in porosity of the surface. Bioleaching can enhance material porosity and alter the mineralogy. This effect can cause an increase in mineral carbonation reactions by overcoming the inherent rate and conversion limitations. In addition the increase in surface porosity in result of biodegradation of the solid waste, can lead to formation of micro and meso-porous structures. Also re-precipitation of altered crystalline phases on the surface of the solid waste can inherently enhance the sorption capacity of certain materials, thus enabling their valorization as sorbents [18].

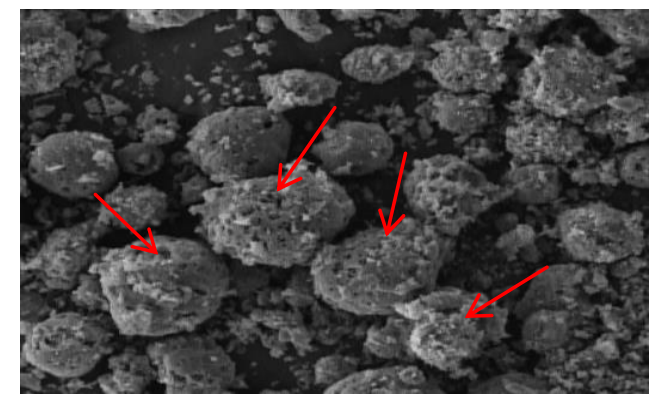

(a)

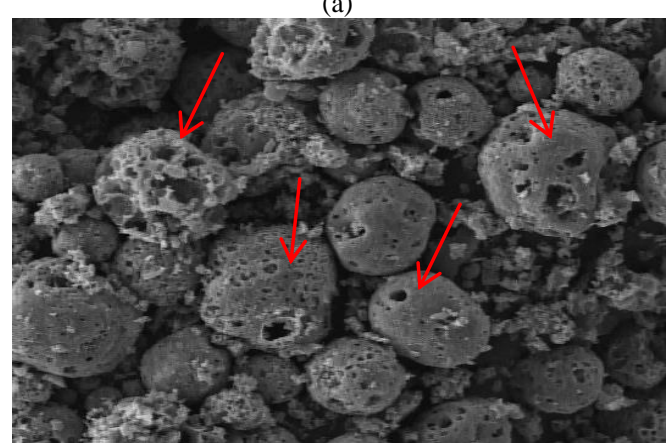

(b)

Fig. 4. FE-SEM of oil-fired ash (a) before bioleaching; (b) after bioleaching.

\section{CONCLUSION}

In this study spent-medium bioleaching of precious metals of vanadium and nickel from an oil-fired ash sample using Aspergillus niger was investigated. The experiments were performed in both Erlenmeyer and bioreactor scales and the results were compared. The achieved recovery yields indicated that in both scales almost total vanadium was recovered, while nickel recovery was lower. In bioreactor experiments, nickel recovery yield was lower in comparison to the shake flask experiments, which it could be due to precipitation of some values of $\mathrm{Ni}$ in presence of high levels of oxalic acid existing in its spent medium. Also it was concluded that using bubble column bioreactor, within a shorter period of time, reaching to the same high vanadium recovery yields was possible.

\section{REFERENCES}

[1] M. Al-Malack, A. Bukhari, O. Al-Amoudi, H. Al-Muhanna, and T. Zaidi, "Characteristics of fly ash produced at power and water desalination plants firing fuel oil," Int. J. Environ. Res, vol. 7, no. 2, pp. 455-466, 2013.

[2] S. L. Tsai and M. S. Tsai, "A study of the extraction of vanadium and nickel in oil-fired fly ash," Resour. Conserv. Recy, vol. 22, no. 3, pp. 163-176, 1998.

[3] R. Moskalyk, and A. Alfantazi, Processing of vanadium: A review," Miner. Eng, vol. 16, no. 9, pp. 793-805, 2003.

[4] S. O. Rastegar, S. M. Mousavi, S. A. Shojaosadati, and R. S. Mamoory, "Bioleaching of $\mathrm{V}, \mathrm{Ni}$, and $\mathrm{Cu}$ from residual produced in oil fired furnaces using Acidithiobacillus ferrooxidans," Hydrometallurgy, vol. 157, pp. 50-59, 2015.

[5] D. Pradhan et al., "Bioleaching kinetics and multivariate analysis of spent petroleum catalyst dissolution using two acidophiles," J. Hazard. Mater, vol. 175, no. 1, pp. 267-273, 2010.

[6] M. Shahrabi-Farahani, S. Yaghmaei, S. M. Mousavi, and F. Amiri, "Bioleaching of heavy metals from a petroleum spent catalyst using Acidithiobacillus thiooxidans in a slurry bubble column bioreactor,' Sep. Purif. Technol, vol. 132, pp. 41-49, 2014.

[7] M. Govarthanan et al., "Bioleaching characteristics, influencing factors of $\mathrm{Cu}$ solubilization and survival of Herbaspirillium sp. GW103 in Cu contaminated mine soil," Chemosphere, vol. 109, pp. 42-48, 2014

[8] T. J. Xu and Y. P. Ting, "Fungal bioleaching of incineration fly ash: Metal extraction and modeling growth kineticks," Enzyme. Microb. Technol, vol. 44, no. 5, pp. 323-328, 2009.

[9] K. M. M. Aung and Y. P. Ting, "Bioleaching of spent fluid catalytic cracking catalyst using Aspergillus niger," J. Biotechnol, vol. 116, no. 2, pp. 159-170, 2005.

[10] A. Bharadwaj and Y. P. Ting, "Bioleaching of spent hydrotreating catalyst by acidophilic thermophile Acidianus brierleyi: Leaching mechanism and effect of decoking," Bioresour. Technol, vol. 130, pp. 673-680, 2013.

[11] G. Natrajan and Y. P. Ting, "Two-step bioleaching and spent medium leaching of gold from electronic scrap material using Chromobacterium violaceum," Adv. Mat. Res, 2013, Trans Tech Publ.

[12] S. Y. Chen and J. G. Lin, "Effect of substrate concentration on bioleaching of metal-contaminated sediment," J. Hazard. Mater, vol. 82, no. 1, pp. 77-89, 2001.

[13] A. Franz, W. Burgstaller, B. Müller, and F. Schinner, "Influence of medium components and metabolic inhibitors on citric acid production by Penicillium simplicissimum," J. Gen. Microbiol, vol. 139, no. 9, pp. 2101-2107, 1993.

[14] H. Grewal and K. Kalra, "Fungal production of citric acid," Biotechnol. $A d v$, vol. 13, no. 2, pp. 209-234, 1995.

[15] L. Karaffa, E.Sándor, E. Fekete, and A. Szentirmi, "The biochemistry of citric acid accumulation by Aspergillus niger (A review)," Acta. Microbiol. Immunol. Hung, vol. 48, no. 3-4, pp. 429-440, 2001.

[16] S. Biswas, R. Dey, S. Mukherjee, and P. C. Banerjee, "Bioleaching of nickel and cobalt from lateritic chromite overburden using the culture filtrate of Aspergillus niger," Appl. Biochem. Biotechnol, vol. 170, no. 7, pp. 1547-1559, 2013.

[17] A. Machuca, G. Pereira, A. Aguiar, and A. Milagres, "Metal-chelating compounds produced by ectomycorrhizal fungi collected frompineplantations," Lett. Appl. Microbiol, vol. 44, no. 1, pp. 7-12, 2007.

[18] Y. W. Chiang et al., "Effects of bioleaching on the chemical, mineralogical and morphological properties of natural and waste-derived alkaline materials," Miner. Eng, vol. 48, pp. 116-125, 2013.

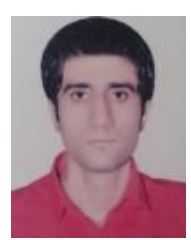

Payam Rasoulnia was born on 1988 in Iran. He is an M.Sc graduate student in chemical engineering-biotechnology from Tarbiat Modares University in Tehran, Iran. He earned his BSC degree in chemical engineering from Sahand University of Technology, Tabriz, Iran.

He has four papers in ISI journals and two papers in national conferences. His research interest lies in the area of environmental biotechnology.

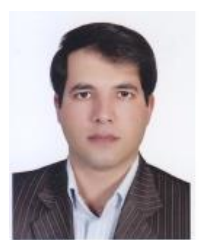

Seyyed Mohammad Mousavi was born in 1978 in Iran He received his $\mathrm{PhD}$ degree in chemical engineering from Sharif University of Technology in 2007, Iran. At the moment, he is an associate professor of industria biotechnology in department of chemical engineering at Tarbiat Modares University.

$\mathrm{He}$ is author of more than 115 ISI papers and 40 national and international conferences. Dr. Mousavi interested in environmental biotechnology especially in bioleaching of hazard wastes, mathematical modeling and simulation of biological phenomena, statistical modelling and optimization of biochemical processes and computational fluid dynamics. 